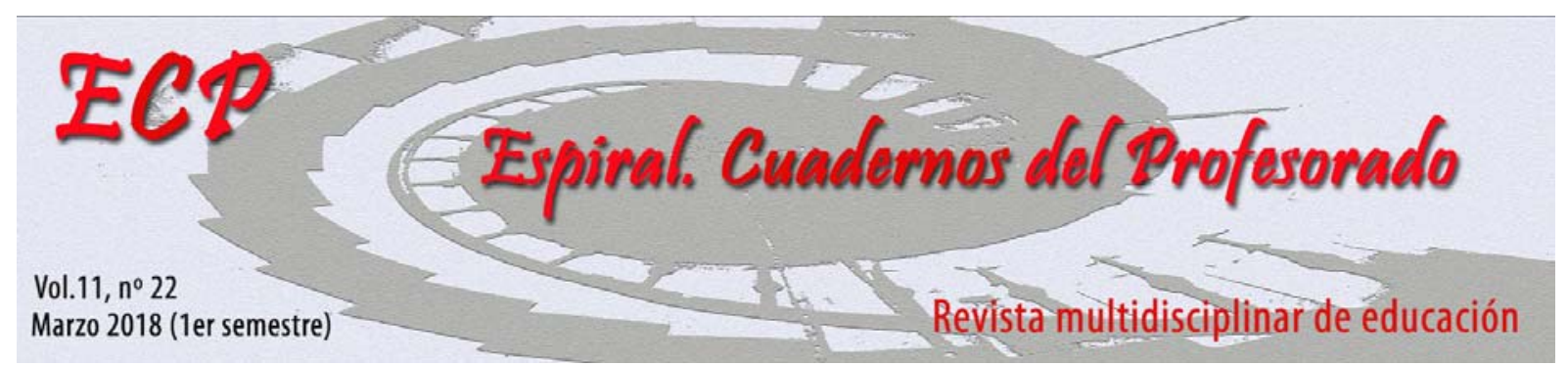

\title{
Fundamentos y características de un modelo inclusivo y de calidad educativa: Comunidades de Aprendizaje
}

\section{Fundaments and features of a educational model of inclusion and quality: Learning Communities}

\section{Francisco Javier Domínguez Rodríguez}

\author{
Asesor de Educación Primaria del Centro del Profesorado Linares-Andújar
}

\begin{abstract}
RESUMEN: En nuestras instituciones educativas la práctica docente está caracterizada por un individualismo y una balcanización absurdos que deja de lado el aprendizaje dialógico. Comunidades de Aprendizaje es un proyecto de transformación de los centros educativos dirigido a la superación del fracaso escolar y la eliminación de conflictos. Este artículo muestra una apuesta por el aprendizaje dialógico mediante la participación de la comunidad educativa y la puesta en práctica de Actuaciones Educativas de Éxito que defiende dicho proyecto, donde el diálogo igualitario se convierte en un esfuerzo común para lograr la igualdad educativa de todo el alumnado del CEIP Santa Teresa Doctora de Linares (Jaén). Parte de la premisa de que la Comunidad Educativa posee recursos, instituciones, agentes... que es preciso identificar, valorar e involucrar en el proyecto educativo de los centros y únicamente esta unión puede hacer posible la educación para todos y el aprendizaje permanente. Mostramos como objetivos de nuestro estudio, conocer los índices de absentismo, resultados académicos, convivencia e inclusión educativa del centro educativo mencionado, a través de la metodología comunicativa crítica, la cual no solamente busca la descripción y/o interpretación de la realidad, sino que pretende ayudar a transformar esa realidad.
\end{abstract}

Palabras clave: Comunidades de Aprendizaje, grupos interactivos, aprendizaje dialógico, Actuaciones Educativas de Éxito.

ABSTRACT: In our educational institutions teaching practice is characterized by an absurd individualism and balkanization that brush aside dialogic learning. Learning Communities is a project to transform schools aimed at overcoming school failure and the elimination of school conflicts. This article shows a commitment to learning dialogue by the participation of the educational community and the implementation of Educative Actions for Success that this project supports, where the equal dialogue turns into a shared effort to achieve educational equality to all the students of Santa Teresa Doctora's Primary School from Linares (Jaén). It starts from the premise that the educational community has resources, institutions, agents ... which are necessary to identify, to value and to involve in the educational project of centres and only this union can make education possible for everybody and lifelong learning. There are showed as aims of this study, know the rate of absenteeism, learning outcomes, school environment and educational inclusion of the school mention, through the questioning and communicative methodology, which not only looks for the description and/or the interpretation of the reality, but instead tries to transform this reality.

Key words: Learning Communities, interactive groups, learning dialogue, Educative Actions for Success.

Domínguez Rodríguez, F. J. (2018). Fundamentos y características de un modelo inclusivo y de calidad educativa: Comunidades de Aprendizaje. Espiral. Cuadernos del Profesorado, 11(22), 2839.

Fecha de recepción: 06/07/2017

Fecha de aceptación: 04/12/2017
Correspondencia:

franciscojavier.dominguez.edu@juntadeandalucia.es 


\section{Introducción}

El proyecto de Comunidades de Aprendizaje pretende cambiar la práctica educativa en los centros para así dar respuesta de forma igualitaria a las nuevas necesidades que se han dado en nuestra población, tras la transformación de una sociedad industrial a una sociedad de la información, junto a muchos más cambios que se están produciendo en la humanidad actualmente. Sin embargo, la sociedad vemos que ha cambiado y, en cambio, si observamos a la escuela actual, podemos ver que básicamente se ha transformado muy poco, exceptuando algunos aspectos en la etapa de Educación Infantil y algunas experiencias en Educación Primaria. En nuestras escuelas sigue aumentando el fracaso escolar y la mala convivencia, y se hace difícil la inclusión educativa. Comunidades de Aprendizaje se presenta como un medio para solucionar estos problemas gracias al aprendizaje dialógico y a la participación de toda la comunidad educativa y agentes externos en el aula. En este artículo se desarrollará el caso de un centro educativo, el CEIP Santa Teresa Doctora de Linares (Jaén), y veremos los resultados que objetivamente está dando dicho proyecto en Andalucía.

"En las Comunidades de Aprendizaje todos los miembros implicados sueñan $y$ construyen la escuela que quieren para sus hijos e hijas con el objetivo de mejorar su educación. La participación de todos y todas es lo que hace posible transformar esa realidad, por más inmutable que parezca, en base a unos principios como el diálogo, la igualdad y la solidaridad” (Elboj, Puigdellívol y Valls, 2002, p. 61).

Será el Centro Especial de Investigación en Teorías y Prácticas Superadoras de Desigualdades (CREA) el que impulse este proyecto, que lo define de la siguiente manera:

"Una Comunidad de Aprendizaje es un proyecto de transformación social y cultural de un centro educativo y de su entorno para conseguir una sociedad de la información para todos y todas las personas, basada en el aprendizaje dialógico, mediante una educación participativa de la comunidad, que se concreta en todos sus espacios, incluida el aula" (Valls, 2000, p. 8; tomado de Elboj et al., 2002).

El desafío de las Comunidades de Aprendizaje consiste entonces, en conseguir dicha escuela inclusiva, aprovechando los recursos que ofrece la propia comunidad, mediante la utilización de recursos y agentes externos e internos provenientes de la propia escuela o de organismos externos colaboradores como asociaciones, ayuntamientos, entidades sin ánimo de lucro... que colaboran con la escuela, todo ello basándose en el aprendizaje dialógico propio de una Comunidad de Aprendizaje.

"Las actuales sociedades multiculturales quedan expuestas a una encrucijada: reconstruyen nuevas formas de vivir juntos (Touraine) en base al diálogo y consenso entre una creciente pluralidad de voces o sufren las consecuencias del conflicto entre diferentes fundamentalismos. La salida a la encrucijada viene de la mano del diálogo y, donde éste se acaba, de su contrario: la violencia” (Flecha, 2004, p. 4).

Se trataba de investigar si se ha dado objetivamente ese cambio que propone el proyecto de Comunidades de Aprendizaje, no solamente en el campo de la inclusión educativa, sino también en el de la mejora de los rendimientos académicos y la calidad educativa.

El análisis del CEIP Santa Teresa Doctora de Linares (Jaén), que es Comunidad de Aprendizaje oficialmente desde el curso 2012/13, nos ayudará a ver los resultados objetivos que producen las Actuaciones Educativas de Éxito que defiende Comunidades de Aprendizaje y el grado de satisfacción que existe entre alumnado, profesorado y voluntariado del centro. Hemos seleccionado este centro por las características peculiares que le rodean, ya que está en una barriada marginal y el $80 \%$ del alumnado es de etnia gitana. En consonancia con el interés de la investigación y con la perspectiva metodológica adoptada, la elección del CEIP Santa Teresa Doctora, como caso único de estudio, viene determinada por los siguientes criterios:

- El centro trabaja el proyecto Comunidades de Aprendizaje desde 2011/12 y aprueban su proyecto en el curso 2012/13.

- El centro ya tiene consolidado el proyecto de Comunidades de Aprendizaje. 
- El centro se encuentra en un contexto de bajo nivel socioeconómico y con una alta presencia de población de etnia gitana.

- El centro es uno de los pioneros en Jaén y Andalucía en abordar su transformación.

\section{Contexto de estudio}

Ofrecemos de una manera general los aspectos más representativos del CEIP Santa Teresa Doctora de Linares (Jaén) para así comprender mejor dicha realidad educativa y con la que nos encontramos en el curso 2011/12. Posteriormente veremos en el estudio si algunos aspectos han cambiado gracias a la transformación del centro en Comunidad de Aprendizaje desde ese curso académico hasta la actualidad.

El CEIP Santa Teresa Doctora de Linares es de titularidad pública y compuesto por 9 unidades (3 de Educación Infantil y 6 de Educación Primaria). Su alumnado pertenece a barriadas que están consideradas como entre las más pobres de la ciudad. Las familias suelen estar desestructuradas y con padres en la cárcel, drogodependientes, o de abandono de hecho. La mayoría de ellos están parados o son pensionistas, y el resto se dedica a la venta ambulante o vive de la recogida de chatarra y de ocupaciones de temporada. Abundan las familias con un nivel cultural bajo con analfabetos funcionales y, por ello, la relación con el centro viene marcada por una cierta desconfianza en la institución escolar, escasamente valorada por madres y padres, posiblemente como consecuencia de creer que la cultura dominante atenta contra la cultura minoritaria. Se manifiesta en la escasa participación de madres y padres en la vida del centro. Nula presencia en las reuniones de tutoría, no recogida de los boletines de calificaciones, baja participación en los procesos electorales, escasa afiliación a la A.M.P.A., etc. Suelen ser frecuentes las visitas a Dirección para exponer quejas contra el centro o el profesorado, muchas infundadas, como consecuencia de dar más credibilidad a lo que dicen niños/as que a lo que dicen maestros/as. El $80 \%$ de su alumnado es de etnia gitana y presenta fuerte déficit en las materias instrumentales. Contaban con un abultado absentismo que alcanzó hasta el $25 \%$, con el consabido perjuicio para el alumnado y, como consecuencia de todo lo anterior, los resultados escolares daban un alto índice de fracaso escolar.

\section{Objetivos e hipótesis de la investigación}

El trabajo de investigación pretendía conocer y analizar la realidad educativa de las Comunidades de Aprendizaje que abogan por una educación más participativa, abierta, integrada y global capaz de proporcionar a todo el alumnado altas expectativas de éxito desde una perspectiva humanista como alternativa a la escuela tradicional. Las Comunidades de Aprendizaje son centros inclusivos comprometidos en desarrollar y potenciar prácticas y principios pedagógicos que combatan el fracaso escolar y la marginación social donde toda la comunidad educativa está comprometida con el proyecto. Por ello, desde esta investigación se marcó el objetivo de conocer la fundamentación teórica y práctica de las Comunidades de Aprendizaje, estableciendo como centro educativo de referencia el CEIP Santa Teresa Doctora de Linares (Jaén). $\quad$ Para ello tratamos de:

- Analizar el CEIP Santa Teresa Doctora de Linares (Jaén) como Comunidad de Aprendizaje.

- Evaluar las posibilidades de transformación de centros educativos tradicionales en Comunidades de Aprendizaje en Andalucía.

También queríamos concretar la viabilidad y los resultados que está dando Comunidades de Aprendizaje y que están transformando la realidad educativa andaluza, centrándonos en aspectos como:

- Mejora de los resultados académicos.

- Cambios en la convivencia escolar del centro.

- Reducción de la tasa de absentismo y abandono escolar en el centro.

- Comunidad de Aprendizaje como modelo de escuela inclusiva. 
- Grado de satisfacción de los agentes internos y externos de la Comunidades de Aprendizaje.

En definitiva, el objeto fundamental de esta investigación es conocer cómo es la transformación de una escuela en una Comunidad de Aprendizaje y las mejoras que se producen con dicha transformación. Deseábamos conocer la experiencia de transformación vivida por las personas, observar los cambios operados y la sostenibilidad de este proyecto en el tiempo, en un centro concreto, además de descubrir las mejoras que se producen en un centro educativo cuando decide transformarse en Comunidad de Aprendizaje.

Aunque se trata de una experiencia concreta, el conocimiento generado, en este sentido, puede ser útil para los centros educativos que planeen transformarse o que ya estén inmersos en el proceso. Queremos que sirva de estímulo y refuerzo para centros educativos que se encuentran en una situación similar al caso propuesto, y logren esta transformación para conseguir que su centro crezca y se beneficie de los resultados que ofrece este proyecto.

Para todo ello, aprovechamos la oportunidad que nos ofrecía el contacto con el CEIP Santa Teresa Doctora de Linares (Jaén) transformado en Comunidad de Aprendizaje hacía varios años. Se trataba de un centro con una tipología peculiar. Inserto en un contexto singular, el contexto de una zona marginal y una población mayormente de etnia gitana; y un centro reputado como innovador, que se mostraba abierto a recibir personas observadoras de su proceso y de sus resultados, y que se mostró dispuesto en todo momento para la realización de esta investigación.

En relación a los objetivos establecidos, en la investigación se plantea una hipótesis de trabajo que será contrastada para confirmarla o refutarla en el proceso de análisis y valoración de resultados. Esta hipótesis es: El proyecto de Comunidades de Aprendizaje transforma el centro educativo y hace que mejore la calidad de la enseñanza: resultados académicos, implicación de agentes educativos internos y externos y se produzca una verdadera inclusión educativa.

\section{Método y participantes}

La metodología utilizada en el presente estudio responde al enfoque comunicativo crítico (Gómez, Latorre, Sánchez, y Flecha, 2006). Dicha metodología no solamente busca la descripción y/o interpretación de la realidad, sino que pretende ayudar a transformar esa realidad. La metodología comunicativa crítica está basada en el diálogo igualitario entre la comunidad científica y las personas cuya realidad sea objeto de investigación, de manera que la propia investigación es el resultado de una pluralidad de voces (Touraine, Wieviorka, y Flecha, 2004). Por todo ello, la investigación se enriquece gracias a las interpretaciones y experiencias de los "protagonistas" de la investigación. Los métodos y técnicas de recogida de datos que se emplearon, así como los participantes del estudio se muestran en la siguiente tabla (Tabla 1).

Tabla 1.

Resumen de las técnicas, códigos y participantes del estudio.

\begin{tabular}{|c|c|c|}
\hline TÉCNICA & CÓDIGO & PARTICIPANTE/S \\
\hline $\begin{array}{l}\text { Observación } \\
\text { participante de orientación } \\
\text { comunicativa }\end{array}$ & $\begin{array}{c}\mathrm{OBS}_{-}^{\mathrm{a}} \text { (Añadir el número de } \\
\text { observación realizada) }\end{array}$ & $\begin{array}{c}\text { Toda la Comunidad de Aprendizaje } \\
\text { del CEIP Santa Teresa Doctora de } \\
\text { Linares (Jaén) }\end{array}$ \\
\hline \multirow{4}{*}{$\begin{array}{l}\text { Entrevistas en profundidad de } \\
\text { orientación comunicativa }\end{array}$} & $\mathrm{EPMV}^{\mathrm{b}}$ & Madre voluntaria \\
\hline & EPMA $^{\mathrm{c}}$ & Voluntaria maestra en activo \\
\hline & $\mathrm{EPD}^{\mathrm{d}}$ & Director \\
\hline & $\mathrm{EPM}^{\mathrm{e}}$ & Maestra $4^{\circ}$ Educación Primaria \\
\hline Grupos de discusión & $\mathrm{GDPV}^{\mathrm{f}}$ & $\begin{array}{l}\text { Tutora } 2^{\circ} \text { EP, voluntaria miembro de } \\
\text { Secretariado Gitano, madre volun- }\end{array}$ \\
\hline
\end{tabular}




\begin{tabular}{|c|c|c|}
\hline comunicativos & & $\begin{array}{c}\text { taria, la maestra de pedagogía } \\
\text { terapéutica del centro y la Jefa de Es- } \\
\text { tudios. }\end{array}$ \\
\hline & $\mathrm{GDA}^{\mathrm{g}}$ & $\begin{array}{c}\text { Cinco alumnos y alumnas de } 6^{\circ} \text { de } \\
\text { Educación Primaria }\end{array}$ \\
\hline $\begin{array}{l}\text { Relato comunicativo de vida } \\
\text { cotidiana }\end{array}$ & $\mathrm{RCVC}^{\mathrm{h}}$ & Jefa de Estudios \\
\hline Análisis documental & $\begin{array}{c}\text { Doc_ }{ }^{\mathrm{i}} \text { (Se añadirá la } \\
\text { abreviatura del documento y } \\
\text { la página donde se encuentra, } \\
\text { si es el caso) }\end{array}$ & $\begin{array}{c}\text { Toda la documentación } \\
\text { perteneciente a la Comunidad de } \\
\text { Aprendizaje del CEIP Santa Teresa } \\
\text { Doctora de Linares (Jaén) }\end{array}$ \\
\hline
\end{tabular}

Nuestro análisis de datos parte de los siguientes elementos que se disponen en un mismo cuadro de análisis:

- Las dimensiones exclusoras y transformadoras: la metodología comunicativa trata de identificar las barreras que impiden la transformación social y las vías de superación de estas barreras; y, en este sentido, aporta estos dos elementos de análisis. La dimensión exclusora hace referencia a las barreras que encuentra la comunidad educativa del CEIP Santa Teresa Doctora para alcanzar una mejora en los resultados académicos, tasas de absentismo, convivencia e inclusión educativa. De igual manera, pretende reflejar las dificultades que se encuentran las familias a la hora de participar en el centro educativo. La dimensión transformadora hace referencia a aquellas prácticas educativas que facilitan, favorecen y promueven el éxito educativo en la comunidad educativa del CEIP Santa Teresa Doctora, y contribuyen a que se alcance una mejora y calidad educativa y promueven la participación activa en la comunidad.

- Las categorías de análisis: la metodología cualitativa aporta estrategias como la codificación y la segmentación de los datos en unidades de análisis, y la agrupación de estas últimas en categorías. El proceso de análisis giró en torno a las dimensiones exclusoras y transformadoras que definimos, que se combinaron con las categorías de análisis construidas que podemos ver en la Tabla 2.

Tabla 2.

Categorización para el proceso de análisis de nuestro estudio.

MEJORA EDUCATIVA

Rendimiento académico

Absentismo escolar

Convivencia

Mejora

Conflictos

aprendizaje

Motivación

\section{INCLUSIÓN EDUCATIVA}

Cooperación entre iguales

Interacciones
Papel de la

Comunidad
Inclusión e integración educativa

ANEAE 


\section{Resultados}

La Comunidad de Aprendizaje del CEIP Santa Teresa Doctora de Linares (Jaén) es un proyecto de transformación que está focalizado en el éxito escolar. Además, el proceso de enseñanzaaprendizaje no recae exclusivamente en manos del profesorado de este centro educativo, sino que depende de la implicación conjunta diferentes sectores: familias, asociaciones y voluntariado, incluyendo al personal docente de este centro educativo. En este sentido, este proyecto inclusivo de transformación social y cultural favorece el éxito escolar, mediante la aplicación de modelos organizativos de aprendizaje dialógico que potencian las interacciones y el diálogo igualitario entre toda la comunidad (profesorado, alumnado, familias, asociaciones y voluntariado) y que han mejorado aspectos como:

- Absentismo escolar: La reducción del abandono es una preocupación compartida en España por todas las administraciones con responsabilidad en esta materia, debido a la alta tasa que presenta el país.

La mayoría de nuestros alumnos/as son de etnia gitana, de nivel socioeconómico y cultural bajo. Los principales problemas del centro eran el bajo rendimiento escolar (la mayoría no continuaban sus estudios de la ESO, sobre todo las niñas), gran absentismo, escasa participación de las familias y diversos conflictos de convivencia. RCVC

La clave está en que el alumnado encuentre un sentido a la escuela y venga motivado cada jornada lectiva, y eso en Comunidades de Aprendizaje se consigue gracias a las Actuaciones Educativas de Éxito que se realizan y a la participación de la comunidad en el centro educativo. El alumnado de cualquier centro debe ver que la escuela es importante para su futuro próximo y si sus familiares acuden a la escuela es que es algo importante.

También es verdad que si un centro te parece agradable, te sientes seguro en él y te gusta la manera de trabajar, no te costará ir cada día a ese centro. Hay que venir con alegría cada día a trabajar, y este equipo docente lo facilita, cosa que echan de menos cuando van al instituto. Aquí están "entre algodones" y siempre pendientes de ellos y dándole mucho cariño por parte de todos, cuando llegan allí es otro mundo bien diferente GDPV V?

Los/as tutores/as de los grupos de este centro educativo son los primeros agentes que actúan justificando o no las faltas de asistencia del alumnado, para ello deben tener un trato directo con las familias y conocer su situación. Una vez que el alumnado está escolarizado, comienzan a ponerse en marcha las medidas de prevención de dicho absentismo, favoreciendo la participación familiar y de asociaciones y entidades de la zona en los procesos educativos, especialmente aquellas que trabajan con el colectivo gitano como es el Secretariado Gitano, desarrollando procesos y procedimientos de evaluación permanente sobre el fracaso y el absentismo escolar, potenciando la atención al alumnado con necesidades educativas especiales asociadas a condiciones sociales desfavorecidas, impulsando actuaciones en materia curricular adaptadas a las realidades de cada centro, implantando de forma coordinada planes y programas dentro del proyecto educativo de zona, atendiendo de manera especial a la población escolar absentista, realizando actuaciones de sensibilización y motivación para la escolarización del alumnado, potenciando la educación y formación de adultos (familiares y voluntariado) para que el alumnado valore el esfuerzo de estudiar a esa edad y la importancia de tener unos estudios para el futuro...

Todavía tenemos que seguir trabajando, ya que hay determinados momentos del año en que el absentismo aumenta. De todas formas ahora si es verdad que te vienen los padres y te justifican esas faltas. GDPV, M1. Vamos por la buena línea pero creo también que hay que mejorar más. Aparte del absentismo, lo que contabilizamos y hacemos estadísticas es sobre la asistencia a clase. Las justificaciones deben ser reales y muchas veces tenemos que estar encima y vamos al Centro de Salud a preguntar si ha ido ese niño a una cita médica ese día, ya que es nuestra obligación y tenemos que estar siempre vigías ante este problema del absentismo. GDPV, M2 
Los resultados de la investigación apuntan que Comunidades de Aprendizaje reduce el absentismo escolar de manera significativa, gracias a la plena participación de la comunidad educativa tanto en la gestión del currículo como en el funcionamiento y la toma de decisiones del centro.

- Convivencia escolar: Uno de los grandes factores que apuestan por la efectividad del proyecto es que lo dialógico provoca la mejora de la convivencia en la escuela y fuera de ella. El diálogo es el camino para lograr esa convivencia.

Ha cambiado mucho la forma de actuar y, aunque es algo inevitable el tener algunos episodios esporádicos de conflicto, están viendo que la única v meior manera de resolverlos es a través del diálogo. EPD.36.

El modelo dialógico apuesta por la resolución de los conflictos a través del diálogo e implica a toda la comunidad en la creación e implantación de normas escolares que ayuden al alumnado a enfrentarse a las distintas dificultades que puedan surgir en la escuela, siendo el principio de validez y no el de autoridad el que defina estas normas de convivencia consensuadas (Grañeras, Díaz-Caneja y Gil, 2011).

Además, hemos puesto en marcha en el centro algunas actuaciones como el Club de los Valientes o la mediación escolar en los recreos, ya que es el momento y lugar donde más "roces" y conflictividad existe. Las normas deben ser pocas para irlas cumpliendo poco a poco y consensuadas por la comunidad. EPD, 37.

Junto a este modelo dialógico de resolución de conflictos, en el centro se establece también el plan de convivencia del centro, el cual tiene en cuenta las características de nuestro alumnado y su entorno, y se basa en los siguientes puntos: La comunicación a través del diálogo para resolver conflictos que ocurran en el día a día, puesta en marcha de manera sistemática de las Actuaciones Educativas de Éxito como grupos interactivos y/o las tertulias dialógicas literarias, cambio de postura ante las familias por parte del profesorado...

La convivencia en el centro ha cambiado, ahora es bastante buena y las causas han sido el profesorado y el director que hay ahora y lo tengo que decir, lo digo donde tenga que decirlo, es estupendo y se dirige siempre a las familias con respeto y las familias se lo devuelven.

Todo el equipo de profesores no solamente hacen de profesores (sonríe) sino también de psicólogos y tratan a los padres y al alumnado muy bien y llegan mucho a ellos y eso evita muchos conflictos. EPMV, 36-37.

También tendremos en cuenta aspectos como la elaboración de normas claras y tener en cuenta todos aquellos aspectos que regulan la convivencia, tales como los sistemas de resolución de conflictos, mecanismos de participación en la toma de decisiones, reparto de poderes y toma de decisiones, la Comisión de Convivencia, mediación escolar, la unión familiar...

Las familias también han cambiado. Antes me decían algunos compañeros que los directores estaban amenazados continuamente y que solamente iban al centro a quejarse de algo que le había pasado a sus hijos y no participaban ni en el Consejo Escolar, órgano colegiado del centro que no conseguía tener la representación mínima de padres en el mismo, y mucho menos participaban en las actividades que organizaba el centro. EPMA, 10.

Podemos extraer de nuestra investigación que con Comunidades de Aprendizaje la convivencia escolar mejora y se previenen y reducen los conflictos, todo ello gracias a que el alumnado, familiares y profesorado tienen la oportunidad de opinar y participar conjuntamente en la gestión de los centros, la creación de normas y resolución de conflictos, mejorando cualitativamente la convivencia tanto en el centro educativo como en toda la comunidad.

- Rendimiento escolar: Una de las metas primordiales de las Comunidades de Aprendizaje es la obtención de buenos resultados académicos por parte de todo el alumnado, ya que los buenos resultados académicos van acompañados, generalmente, de la superación de las desigualdades y la inclusión educativa. Se fundamentan en las teorías y actuaciones más 
relevantes que han demostrado tener éxito educativo, es decir, en las Actuaciones Educativas de Éxito.

El proyecto de Comunidades de Aprendizaje es diferente a otros proyectos, es muy completo y consiste en llegar a conseguir altas expectativas para todo el alumnado, es decir, conseguir lo mejor que podamos para nuestro alumnado. Creo que la diferencia con otros centros es la apertura a toda la comunidad y la puesta en práctica de las Actuaciones Educativas de Éxito en el centro: grupos interactivos, tertulias dialógicas... que no tienen otros centros y pienso que debían ponerlas en acción. Con las tertulias hemos acercado a los niños la literatura, música, arte... cosa que, si no fuera de esta manera quizás no hubiera llegado hasta ellos con tanta intensidad, pero lo que más me gusta de todo es el hecho de que los padres vengan y vean a sus hijos dentro de las aulas y que participen y muestren interés. Hemos conseguido que los padres colaboren con nosotros y vean lo importante que es la educación de sus hijos. Los niños están deseando que lleguen a la clase, también tienen interés de que sus padres vengan y es que los buscan. Otro aspecto que no tienen otros centros es el nivel de ayuda que se da entre el alumnado dentro del aula especialmente a través de los grupos interactivos. GDPV. M1.

Entre las Actuaciones Educativas de Éxito más demandadas por el alumnado y que más gustan al profesorado y a la comunidad educativa están los grupos interactivos, ya que aprenden juntos cooperativamente y de una manera más efectiva en el aula al realizar actividades de manera simultánea en el aula.

Pues me gustan mucho sobre todo los grupos interactivos porque me gusta estar en grupo y aprender todos juntos.

Cuando estamos en los grupos interactivos estamos juntos y cuando alguien no sabe algo nos ayudamos y es mejor. Los maestros van pasando por los grupos y nos ayudan cuando hay algo que no entendemos ninguno del grupo, además de ayudarnos en los grupos también te escuchan cuando tienes algún problema, no te dejan. GDA, A y H.

Sobre los efectos en el rendimiento académico del alumnado asociados a la organización inclusiva del aula (INCLUD-ED, 2011, p. 48-49) podemos señalar que cuando utilizamos el aprendizaje dialógico a través de un agrupamiento heterogéneo de grupos interactivos el rendimiento académico mejora, tanto en el alumnado que obtiene buenos resultados, como en el que tiene un menor nivel de aprendizaje, ya que éste último se beneficia del ritmo de trabajo de los grupos más avanzados.

También el alumnado con discapacidades o que presenta dificultades en el aprendizaje obtiene mejores resultados académicos y desarrolla una mayor autoestima en clases integradas que separado del grupo de referencia y no produce efectos negativos en el rendimiento de sus compañeros y compañeras, proporcionando nuevas oportunidades de aprendizaje para todos.

...favorecen bastante el aprendizaje además que favorecen un aprendizaje diría cooperativo, porque ya los niños han cogido la mecánica y cuando alguno acaba antes ya no tienes nada más que mirarlo y decirle por qué no ayudas a tu compañero o compañera.

Ellos saben perfectamente los alumnos que suelen quedarse más atrás y se levantan, se van hacia su lado y le ayudan. EPMA, 24-25.

En la Tabla 3 pueden verse los resultados positivos que se han alcanzado en el CEIP Santa Teresa Doctora, obteniéndose una puntuación durante el curso 2015/16 superior a los centros de la zona y de la media andaluza. Lo que lleva a confirmar lo expuesto anteriormente: La composición cultural del alumnado no es lo que influye en los resultados académicos, sino que lo que influye es la aplicación o no de Actuaciones Educativas de Éxito. 
Tabla 3.

Resultados Prueba Escala desde el curso 2011/12 hasta el curso 2015/16.

\begin{tabular}{|c|c|c|c|c|c|}
\hline \multicolumn{6}{|c|}{ Competencia en Comunicación Lingüística } \\
\hline \multirow{2}{*}{$\begin{array}{l}\text { Categorías } \\
\text { (Máxima puntuación: } 6 \text { puntos) }\end{array}$} & \multicolumn{5}{|c|}{ Curso académico } \\
\hline & $11 / 12$ & $12 / 13$ & $13 / 14$ & $14 / 15$ & $15 / 16$ \\
\hline Comprensión lectora & 3.0 & 4.2 & 5.0 & 5.6 & 5.8 \\
\hline Expresión escrita & 2.0 & 2.4 & 4.9 & 3.2 & 4.8 \\
\hline \multicolumn{6}{|c|}{ Competencia Matemática } \\
\hline \multirow{2}{*}{ Categorías } & \multicolumn{5}{|c|}{ Curso académico } \\
\hline & $11 / 12$ & $12 / 13$ & $13 / 14$ & $14 / 15$ & $15 / 16$ \\
\hline Organizar, comprender problemas & 4.0 & 3.7 & 5.7 & 5.8 & 5.9 \\
\hline Expresión matemática & 2.0 & 4.3 & 5.6 & 5.8 & 5.5 \\
\hline Plantear y resolver problemas & 2.0 & 4.1 & 5.5 & 5.7 & 5.7 \\
\hline
\end{tabular}

Fuente: Agencia Andaluza de Evaluación Educativa. Consejería de Educación de Andalucía.

- Inclusión educativa: El centro educativo objeto de estudio es inclusivo por defender la equidad y calidad educativa para todo el alumnado si hacer excepciones o distinciones. Además, lucha contra la exclusión y separación del alumnado. El modelo de inclusión consiste en crear agrupaciones heterogéneas, de alumnado diverso, y en la reorganización de los recursos para apoyar al alumnado dentro de esos grupos (INCLUD-ED Consortium, 2015).

Ahora con respecto al grupo que tuve anteriormente estoy cambiando en aspectos para mejorar en el tema de la inclusión. Tuve algunos niños absentistas y de NEAE que tenían casi dos años de desfase con respecto al grupo y cuando empezábamos con grupos interactivos ellos no se adaptaban y tenía que darle fichas adaptadas a su nivel. Aunque venían los maestros de refuerzo y le daban éste dentro de la clase, al final el resto del alumnado sabían que eran diferentes al resto de la clase porque venía un maestro a darles clase a ellos solos y su tutora estaba con el resto del grupo haciendo otras cosas.

Ahora todo es diferente.

El maestro de refuerzo no está pegado la hora entera a un alumno o alumna con Necesidades Específicas de Apoyo Educativo o con dificultades de aprendizaje, sino que va pasando por todos los grupos interactivos y el apoyo se hace al grupo y, en situaciones puntuales, es cuando se dirige al alumno o alumna que requiera un apoyo puntual dentro de la clase. Ahora ya no se ve a estos niños como un ente aparte. Ahora se da la verdadera inclusión. EPM4,30-33

Las aulas se organizan para que en las Actuaciones Educativas de Éxito que se llevan a cabo, especialmente en los grupos interactivos, se produzcan interacciones de ayuda. El alumnado que termina antes las tareas ayuda a este tipo de alumnado y se dan cuenta de que recuerdan y consolidan mejor los aprendizajes, lo que repercute en un beneficio propio y en la promoción de valores solidarios en el aula, olvidando los competitivos.

A veces tú piensas algunas cosas de una persona o compañero y no es así, porque las apariencias engañan. Aprendemos a aceptar a todos los compañeros de la clase, relacionándonos con compañeros que de otra manera no me relacionaría nunca con algunos de ellos. GDA, J.

El apoyo de los compañeros y compañeras en los grupos interactivos se presenta como un beneficio no solamente para el alumnado con dificultades, sino que favorece a todos, lo que hace de las Comunidades de Aprendizaje, centros que permiten aprovechar mucho más un capital humano que 
está presente en ellos - el propio alumnado, el profesorado, las familias y la comunidad - en beneficio de una escuela más inclusiva (Molina, 2015, p. 389).

En el CEIP Santa Teresa Doctora cada vez hay más familiares y voluntariado que participa activamente, lo que hace que el alumnado de estas características se sienta más atendido y pueda mejorar, ya que es consciente de que está aprendiendo y lo está haciendo igual que sus compañeros, hecho que aumenta su autoestima y motivación.

\section{Conclusiones}

En cuanto al absentismo escolar, los resultados de la investigación apuntan que Comunidades de Aprendizaje reduce el absentismo escolar de manera significativa, gracias a la plena participación de la comunidad educativa tanto en la gestión del currículo como en el funcionamiento y la toma de decisiones del centro. A raíz de la investigación hemos podido concluir que:

1. Existe relación entre alumnado absentista y rendimiento/aprendizajes del resto de compañeros/as de un mismo grupo-aula. Solución: Aceleración del aprendizaje y pedagogía de máximos.

2. Se produce una reducción del absentismo y aumento de la matriculación gracias a un clima de disciplina, actitud y formación del profesorado e implicación de padres en el centro educativo.

3. Las causas principales del absentismo en nuestro centro eran la falta de apego de las familias al centro, visión negativa de la escuela y falta de relación escuela y familia.

Si nos centramos en los resultados de la investigación con respecto a la convivencia escolar muestran que: Comunidades de Aprendizaje mejora la convivencia escolar dado que desarrolla un modelo dialógico de resolución de conflictos donde participa toda la comunidad educativa en el consenso de la norma, junto con su papel en grupos interactivos, tertulias dialógicas, comisiones mixtas... Algunos de los aspectos a destacar son:

1. El modelo disciplinar de resolución de conflictos no elimina todos los problemas de convivencia en el centro educativo.

2. El modelo experto de resolución de conflictos (mediador) no elimina todos los problemas de convivencia en el centro educativo.

3. El modelo comunitario o dialógico de resolución de conflictos ayuda a prevenir conflictos y resolver problemas de convivencia en el centro educativo.

4. Hay que consensuar la norma entre todos y centrarnos en una, no en una interminable lista de normas impuestas por la autoridad.

5. Las actuaciones educativas que promueven la convivencia en el centro son la formación de familiares, los grupos interactivos, creación de comisiones mixtas de trabajo, las asambleas del grupoclase y el consenso de la norma.

Los resultados de la investigación referentes a los resultados académicos, muestran que: Comunidades de Aprendizaje mejora los resultados académicos del alumnado en pruebas internas y externas, en gran parte gracias a la organización del aula en grupos interactivos y por la realización de las tertulias dialógicas literarias de manera sistemática en el aula. Será importante destacar que:

1. Los efectos asociados a la organización inclusiva del aula a través de los grupos interactivos mejora el aprendizaje en el centro educativo.

2. La realización de tertulias literarias dialógicas en el centro educativo no solamente constituyen una oportunidad de acercarse a la

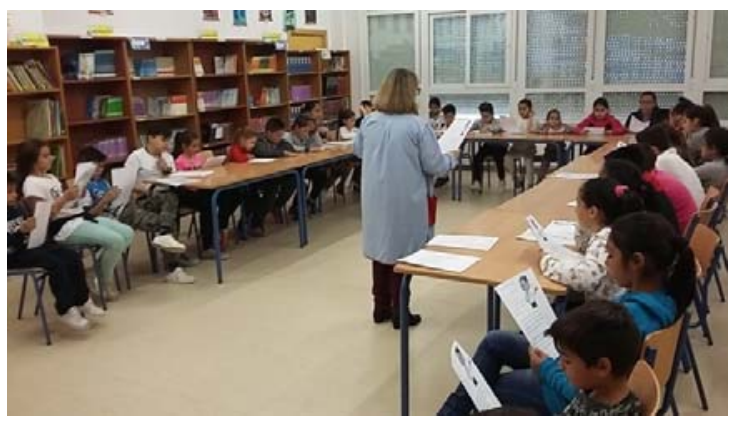

Figura 1. Tertulia dialógica literaria en el CEIP Santa Teresa Doctora de Linares (Jaén). Nota: Elaboración propia. 
literatura clásica. $\mathrm{Al}$ fomentar el diálogo e intercambio de interpretaciones derivadas de la lectura de la obra, se construye conocimiento compartido a partir del texto leído, se refuerza una lectura crítica y la comprensión lectora, mejoran habilidades cognitivas en relación con la lectura (Figura 1).

En cuanto a la inclusión educativa, podemos concluir diciendo que los resultados de la investigación muestran que los centros Comunidades de Aprendizaje son verdaderos centros inclusivos gracias a la participación democrática de la comunidad, las agrupaciones del alumnado, el trabajo colaborativo del profesorado y a la realización de apoyos y refuerzos dentro del aula. Señalamos que:

1. Las actividades que fomentan la inclusión del alumnado en el centro son principalmente los grupos interactivos y una nueva agrupación del alumnado en las aulas del centro.

2. El trabajo colaborativo entre el equipo docente y la participación de voluntariado y familiares ayuda a la inclusión educativa en el centro.

3. Los apoyos y refuerzos al alumnado se realizan dentro del aula para una verdadera inclusión educativa en el centro.

Para terminar las conclusiones, podemos terminar diciendo que en el CEIP Santa Teresa Doctora de Linares (Jaén) se han reducido las tasas de abandono escolar y/o absentismo, ha mejorado la convivencia a nivel de centro y de aula, los resultados académicos y existe una verdadera inclusión educativa. Pensamos que existen prácticas en este centro educativo que pueden transferirse a la escuela tradicional y que repercutirían positivamente en el rendimiento académico y en la interacción social del alumnado, así como a todos los centros que ya están transformados en Comunidad de Aprendizaje:

- Trabajo en equipo (implica desarrollar unos hábitos de convivencia, comunicación, decisión, responsabilidad, valoración...).

- Diálogo igualitario.

- Inclusión de las familias (la comunicación entre la familia y el profesor en un diálogo entre iguales conducente a promover y facilitar el aprendizaje del alumnado dando el soporte necesario a las familias, es un elemento clave para mejorar los problemas que subyacen a lo largo de la vida escolar).

- Enseñanza compartida entre el equipo docente.

- Apoyo dentro del aula.

- Metodologías flexibles y activas como grupos interactivos, tertulias dialógicas...

\section{Referencias}

Elboj, C., Puigdellívol, I., Soler, M., y Valls, R. (2002). Comunidades de aprendizaje: Transformar la educación. Barcelona: Graó.

Flecha, R. (2004). Conocimiento e identidad. Voces de grupos culturales en la investigación social. Barcelona: Hipatia Editorial.

Gómez, J., Latorre, A., Sánchez, M., y Flecha R. (2006). Metodología comunicativa crítica. Ed. El Roure. Barcelona.

Grañeras, M., Díaz-Caneja, P., y Gil, N. (Coords.). (2011). Actuaciones de éxito en las escuelas europeas. Madrid: Secretaria General Técnica. Subdirección General de Documentación y Publicaciones.

INCLUD-ED (2011). Actuaciones de éxito en las escuelas europeas. Madrid: Ministerio de Educación, Cultura y Deporte. Subdirección General de Documentación y Publicaciones.

INCLUD-ED (2012). Final Report. En: http://creaub.info/included/wp-content/uploads/2010/12/D25.2_FinalReport_final.pdf (Consultado el 24 de agosto 2017).

INCLUD-ED Consortium (2015). Successful Educational Actions for Inclusion and Social Cohesion in Europe. Netherland: Springer International Publishing.

Molina, S. (2015). La inclusión del alumnado con necesidades educativas especiales en Comunidades de Aprendizaje. Intangible Capital, 11(3), 372-392. DOI: /10.3926/ic.642 
Touraine, A., Wieviorka, M., y Flecha, R. (2004). Conocimiento e identidad. Voces de grupos culturales en la investigación social. Barcelona: Hipatia Editorial.

Valls, R. (2000). Tesis doctoral: Comunidades de Aprendizajes: una práctica educativa de aprendizaje dialógico para la sociedad de la información. Universidad de Barcelona, Barcelona.

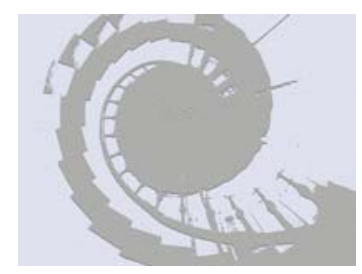

